\title{
Infrared-based sensor system for contactless detection of wetness and ice on surfaces
}

\author{
Lakshan Tharmakularajah ${ }^{1}$, Jakob Döring ${ }^{1}$, Leonard Friedrich ${ }^{1}$, Karl-Ludwig Krieger ${ }^{1}$ \\ ${ }^{1}$ Institute of Electrodynamics and Microelectronics (ITEM.ae), University of Bremen, Germany
}

\{lakshan, doering, friedrich, krieger\}@item.uni-bremen.de

\begin{abstract}
In order to differentiate between a wet and a dry road surface, the water film height is measured by using an infrared-based sensor system. With the help of different wavelengths it is also possible to distinguish between ice and water. In this article, a sensor system for determination of the physical state of water on different surfaces with infrared LEDs and one photodiode is presented. This shall serve as a basis for the calculation of the road condition. Here, emitted and reflected signals of five different infrared emitters are acquired with an infrared receiver. In commercial sensors this method (with one emitter) is used to determine a distance. At a constant distance, different intensities for the reflected signal are measured depending on the color and texture of the surface. Similar sensor systems work with a broadband light, usually a halogen lamp, and several photodiodes. This type is more cost-intensive and not robust enough for the desired application. The transmission spectrum of water shows that light is absorbed little at a wavelength of $900 \mathrm{~nm}$ and strongly at a wavelength of about $1400 \mathrm{~nm}-1500 \mathrm{~nm}$. Therefore, the intensity depends not only on the material surface, but also on the wavelength of the transmitting and receiving diode. In order to distinguish between ice and water, the wavelengths $1450 \mathrm{~nm}$ and $1550 \mathrm{~nm}$ are compared, which is shown in this article by the measurements. In ice, the infrared light is more strongly absorbed at $1550 \mathrm{~nm}$ and less absorbed at $1450 \mathrm{~nm}$.
\end{abstract}

Keywords: optical measurement method, infrared light sensor, ice and water detection, contactless sensor, road condition sensor

\section{Introduction}

Common causes of personal injury in traffic are poor road conditions [1]. Between 2014 and 2017, slippery roads caused around 45900 accidents in Germany due to rain, snow and ice. That is around $3.5 \%$ to $4.1 \%$ of all accidents involving personal injury in Germany and the trend is rising. Drivers regularly lose control of their vehicle due to slippery roads. As a result, the demand for technologies that reliably detect critical road conditions of this kind is growing. Environmental detection and knowledge of road conditions are becoming increasingly important as automation and autonomous driving increase in the automotive sector. This is necessary for autonomous driving in demanding weather conditions. The environmental sensors already installed in vehicles are not sufficient, as they can only indirectly determine road conditions. For example, the rain-light sensor can only be used on a wet road surface when it is raining. Afterwards, the road is still wet but the sensor cannot be used to determine the road condition. There is an approach of indirect road condition monitoring with the use of capacitive sensors in the wheel arch liner [2]. To monitor the road surface, directly visible image sensors, infrared image sensors and millimeter-wave radar sensors are used [3, 4]. These sensors are complex and partly not suitable for vehicles. Therefore, the motivation is to determine the water film height with a novel sensor.

The technology for the determination of ice and water using infrared radiation is already known [4]. However, expensive spectrometers are used for the analyses. In [5], three photodiodes were used as a receiver and a broadband $20 \mathrm{~W}$ halogen lamp was used as the light source for the measurements. This method (with one emitter and receiver) is used to determine a distance with commercially available sensors [6]. In this approach a broadband photodiode and five selected LEDs with defined wavelengths are used as a light source.

\section{Infrared-based sensor system}

The aim of the sensor system presented here is to detect wetness or water on a surface and to record the height of the water film. Furthermore, only one broadband photodiode is used. For the 
selection of suitable LEDs, the wavelengths in the transmission spectrum of water and the absorption spectrum of ice are considered.

A theoretical approach implies that due to the presence of a water film on a surface, the light intensity of the wavelength with a high transmittance decreases less than the intensity with a low transmittance [4]. In [7] the comparison of wavelengths $1.4 \mu \mathrm{m}$ and $1.5 \mu \mathrm{m}$ for ice detection is described. From the absorption spectrum of ice, it can be concluded that the wavelengths up to $1.4 \mu \mathrm{m}$ are absorbed less than the wavelength at $1.5 \mu \mathrm{m}$. The transmittance spectrum (blue solid line) and the absorption spectrum (red dashed line) are shown in Fig. 1. By comparing the transmittance of water and the absorbance of ice, it can be shown that in particular the wavelengths at $1.4 \mu \mathrm{m}$ and $1.5 \mu \mathrm{m}$ can give information about the state of aggregation of water.

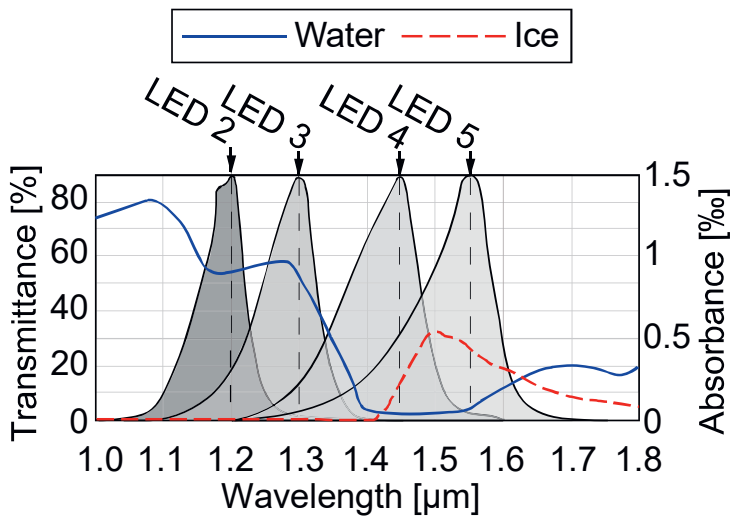

Fig. 1: $\quad$ Transmission spectrum of water (blue solid line) [7] and absorption spectrum of ice (red dashed line) [8] with the selected four LEDs.

To build the sensor system, five different LEDs are selected as the light source and one photodiode is used as the receiver. LED 1 lies outside the shown transmission spectrum in Fig. 1, this has an emission spectrum of $0.92 \mu \mathrm{m}$ to $0.97 \mu \mathrm{m}$ and the highest intensity is at $0.945 \mu \mathrm{m}$. From the emission spectra of LED 2 to 5 it can be seen that the spectra overlap.

Tab. 1: Comparison of the main features of the selected LEDs

\begin{tabular}{|l|r|r|r|}
\hline & Designation & $\begin{array}{c}\text { Wave- } \\
\text { length } \\
{[\mu \mathrm{m}]}\end{array}$ & $\begin{array}{c}\text { Radiant } \\
\text { power } \\
{[\mathrm{mW}]}\end{array}$ \\
\hline LED 1 & $\mathrm{L} 9338$ & 0.945 & 15.0 \\
\hline LED 2 & $\mathrm{L} 13072$ & 1.200 & 5.0 \\
\hline LED 3 & $\mathrm{L} 12771$ & 1.300 & 3.1 \\
\hline LED 4 & $\mathrm{L} 10660$ & 1.450 & 2.8 \\
\hline LED 5 & $\mathrm{L} 12509$ & 1.550 & 3.8 \\
\hline
\end{tabular}

The LEDs are arranged in a circle with the same distance to the photodiode, so that they have a similar influence. To extract the signals of the LEDs, in the signal of the photodiode, the LEDs are pulsed with different frequencies. This sensor system is shown in Fig. 2. The selected photodiode (G12180-030) is used as receiver. The photodiode consists of the semiconductor material indium gallium arsenide also referred to as InGaAs photodiode. This has a round, light-sensitive surface with a diameter of $0.3 \mathrm{~mm}$, which reacts in a wavelength range of $0.9 \mu \mathrm{m}$ to $1.7 \mu \mathrm{m}$.

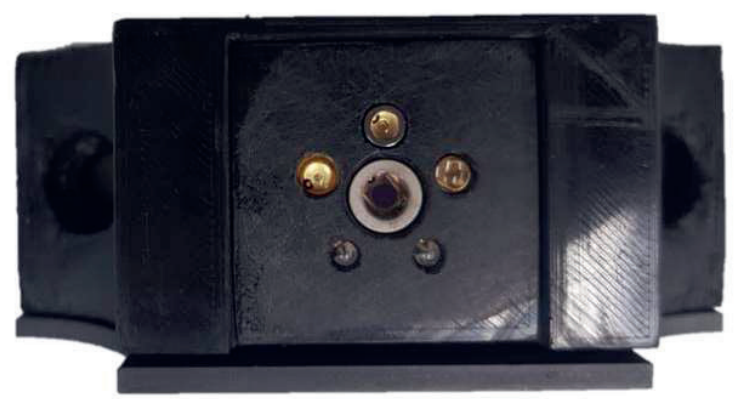

Fig. 2: Infrared-based sensor system with five LEDs and one photodiode.

\section{Measurement setup}

In this section, the setup of the measurement environment is presented. For the measurements, a test bench was set up which sprays a water film with a defined height in $1 \mathrm{~mm}$ steps onto a surface. The test bench shown in Fig. 3 can be controlled in horizontal and vertical direction with a resolution of $1 \mathrm{~mm}$. In order to reproducibly generate water film heights with an accuracy of $1 \mathrm{~mm}$, a strain gage balance was implemented under the surface. The strain gage balance measures the weight, while a pump applies a fine spray to the surface. According to equation (1), the given density $\rho$ of the water allows the weight $m$ to be calculated into the volume

$$
V=\frac{m}{\rho} .
$$

By the calculated volume $V$ and a given surface of a tile $a \cdot b$ the water film height

$$
W F H=\frac{V}{a^{2}}
$$

can be determined. In this case $a=b$. Therefore, the area of the surface is $a^{2}$. The white and black tiles measuring $20 \mathrm{~cm} \times 20 \mathrm{~cm}$ are used for the tests. Boundaries were placed on the sides of the surfaces to prevent sprayed water from overflowing. 


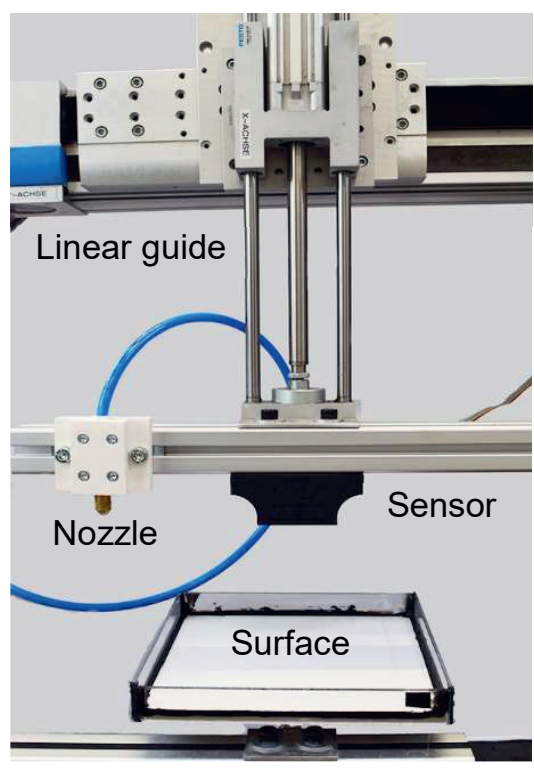

Fig. 3: Test bench for defined water film heights.

\section{Experimental study}

In this section the performed experiments and results are presented. In order to be able to make tangible statements on the individual changed parameters of the distance and the surface, a protection against extraneous light is used.

First of all, two differently colored surfaces, a white and a black tile, were examined. At first, the measurements were carried out on a dry surface to investigate the influence of the different surface properties. In this case, the surfaces in a dry state consequently had a water film height of $0 \mathrm{~mm}$ were investigated. The intensities of the individual LEDs were measured from $5 \mathrm{~cm}$ to $10 \mathrm{~cm}$ in $1 \mathrm{~cm}$ steps.

Subsequently, wet measurements were carried out. A water film of $1 \mathrm{~mm}$ to $10 \mathrm{~mm}$ was measured in $1 \mathrm{~mm}$ steps at a distance of $5 \mathrm{~cm}$ on the white surface. Finally, on the white surface ice with a thickness of $1 \mathrm{~mm}$ was measured with the sensor system and the results were compared with those of water.

\section{A. Distance and intensity analysis}

The measurements were performed at a distance, between the sensor and the surface, of $5 \mathrm{~cm}$ to $15 \mathrm{~cm}$ in $1 \mathrm{~cm}$ steps, which are shown in Fig. 4 and Fig. 6. For all steps 5 measurements with $10 \mathrm{~s}$ each were performed. The measurement data were acquired with a sampling rate of $500 \mathrm{kHz}$ and then averaged. The light emitted by the LEDs is reflected by the two different surfaces and converted into a current by the photodiode. A voltage is generated by a transimpedance converter, which is digitally acquired. At a distance of $5 \mathrm{~cm}$ to $15 \mathrm{~cm}$ from the surface, different intensities of each LED can be observed.
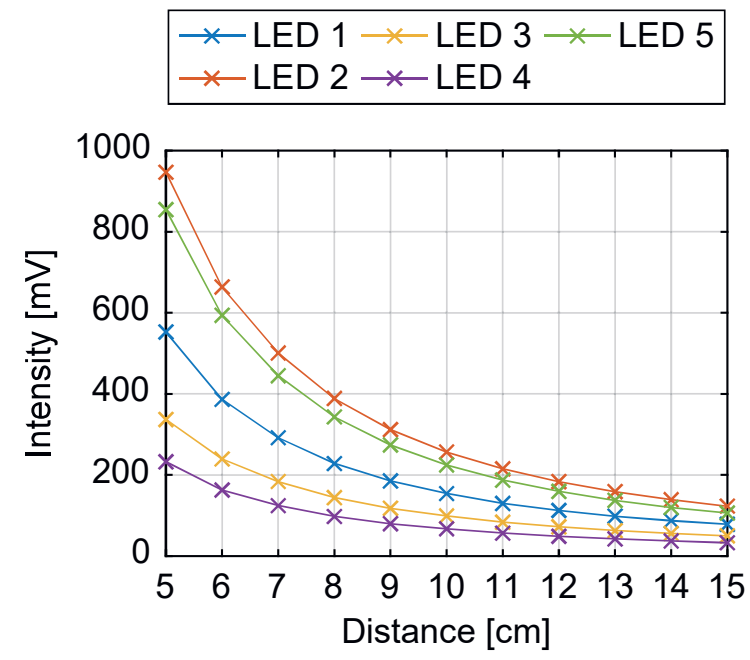

Fig. 4: Intensity at different distances from $5 \mathrm{~cm}$ to $15 \mathrm{~cm}$ in $1 \mathrm{~cm}$ steps with the white surface.

On the one hand this can be attributed to the different spectral sensitivity of the photodiode and on the other hand to the different radiant powers of the LEDs listed in Tab. 1. LED 1 has the highest radiant power, but it has the shortest wavelength. This results in increased reflections at the surface molecules. Due to the coarseness of the tile, the light beams of LED 1 are not reflected uniformly but scattered. This means that fewer light rays penetrate into the sensitive area of the photodiode and therefore the intensity is lower. In addition, the spectral transmission of the protective glass and the sensitivity of the photodiode contribute to LED 2 having the highest intensity value and LED 4 the lowest intensity value.
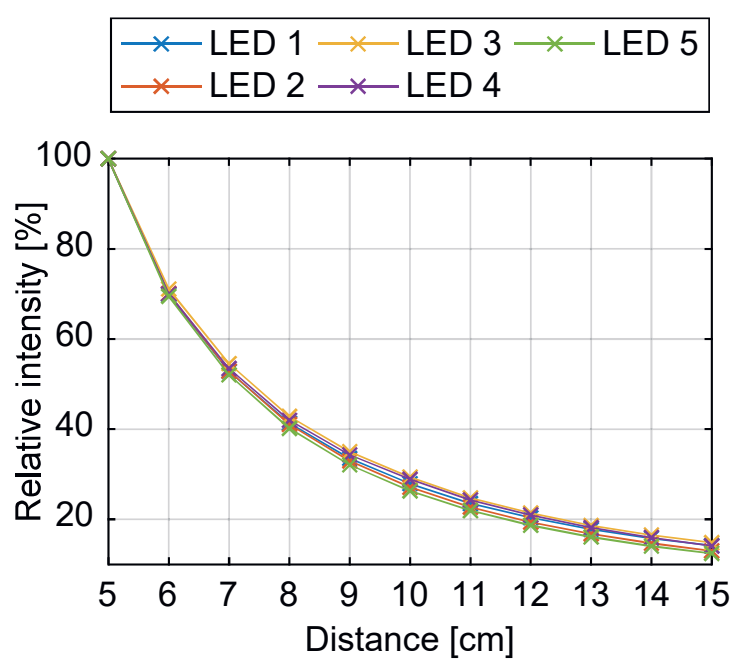

Fig. 5: Relative intensity decrease at distances of $5 \mathrm{~cm}$ to $15 \mathrm{~cm}$ with white surface. 
By observing the course of the intensities $I$ with increasing distance $r$, it is possible to recognize that these follow the inverse-square law of distance. The inverse-square law describes the decrease of a physical quantity with increasing distance to the source or transmitter [9].

$$
I \sim \frac{1}{r^{2}}
$$

This behavior is more clearly recognizable by the representation of the relative intensity to the smallest distance on a white surface. Fig. 5 shows that the intensities of all LEDs decreases quadratically with increasing distance. The intensity is reduced to a quarter of the initial intensity when the distance is doubled from $5 \mathrm{~cm}$ to $10 \mathrm{~cm}$. Analogous to the measurements with the white surface, the measurements and results with the black surface are considered in the following. Fig. 6 shows the measured intensities at different distances with the black surface. Here it can be seen that the order of the intensity of the individual LEDs is almost identical for white and black tiles. The light of LED 2 is reflected most strongly and the light of LED 4 most weakly from the black surface back to the photodiode. LED 1 and LED 3 have an almost identical behavior.

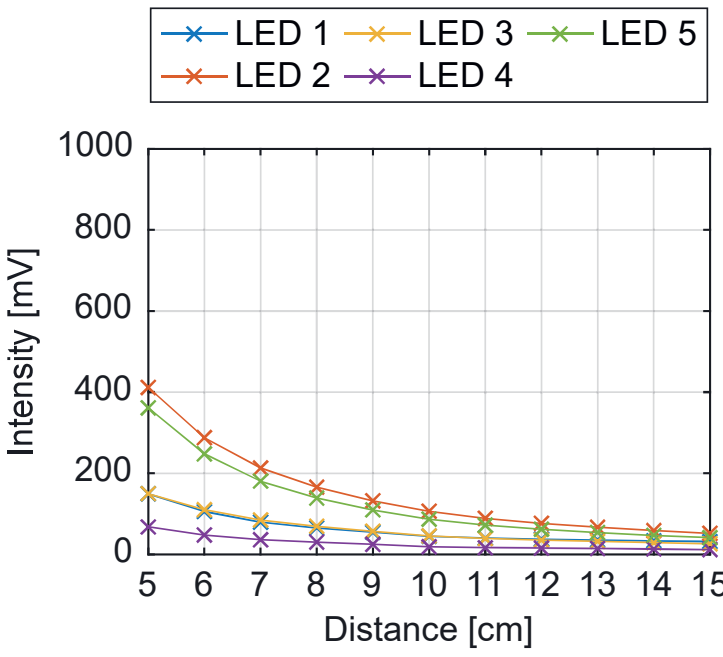

Fig. 6: Intensity at different distances from $5 \mathrm{~cm}$ to $15 \mathrm{~cm}$ in $1 \mathrm{~cm}$ steps with the black surface.

The direct comparison of the intensity values between the white and black surface at a distance of $5 \mathrm{~cm}$ shows a difference. This difference is due to the increased reflectivity of the white surface and the increased absorption of the black surface. A quantification of the values at a distance of $5 \mathrm{~cm}$ for the white and black surface and the resulting decrease in intensity are given in Tab. 2. The intensity of LED 2, 3 and 5 are absorbed almost uniformly by the surface of the black tile. LED 1 and 4 show a decrease in intensity of about $70 \%$. The wavelength-dependent refractive index and the absorption behavior of the black tile could be decisive for this.

Tab. 2: Intensity decrease between the white and black tiles

\begin{tabular}{|c|c|c|c|}
\hline & $\begin{array}{c}\text { White } \\
\text { tile } \\
{[\mathrm{mV}]}\end{array}$ & $\begin{array}{c}\text { Black } \\
\text { tile } \\
{[\mathrm{mV}]}\end{array}$ & $\begin{array}{c}\text { intensity } \\
\text { decrease } \\
{[\%]}\end{array}$ \\
\hline LED 1 & 552.51 & 149.72 & 72.90 \\
\hline LED 2 & 946.67 & 411.48 & 56.53 \\
\hline LED 3 & 336.78 & 149.80 & 55.52 \\
\hline LED 4 & 232.59 & 67.82 & 70.84 \\
\hline LED 5 & 854.55 & 361.33 & 57.72 \\
\hline
\end{tabular}

\section{B. Investigation of water film heights}

The measurements are carried out at a fixed distance of $5 \mathrm{~cm}$ between sensor and surface. A water film layer of $1 \mathrm{~mm}$ to $10 \mathrm{~mm}$ was applied to the substrate in $1 \mathrm{~mm}$ steps. The measured intensity values of the individual LEDs for water film height from $0 \mathrm{~mm}$ to $10 \mathrm{~mm}$ with a sensor distance of $5 \mathrm{~cm}$ are shown in Fig. 7 . The water film height of $0 \mathrm{~mm}$ describes the dry condition of the surface. The measurement with a water film height of $1 \mathrm{~mm}$ shows that the intensity of the individual LEDs decrease. This decrease in intensity can be explained by the light beam path described in [10]. The light beam is not only reflected on the surface of the ground, but first partially reflected on the water surface. The transmitted light beam is partly absorbed by water. The strength of the absorption is different for each wavelength of the LEDs. The light beam is then reflected at the surface of the substrate, absorbed again by the water, refracted at the transition between water and air and received by the photodiode.

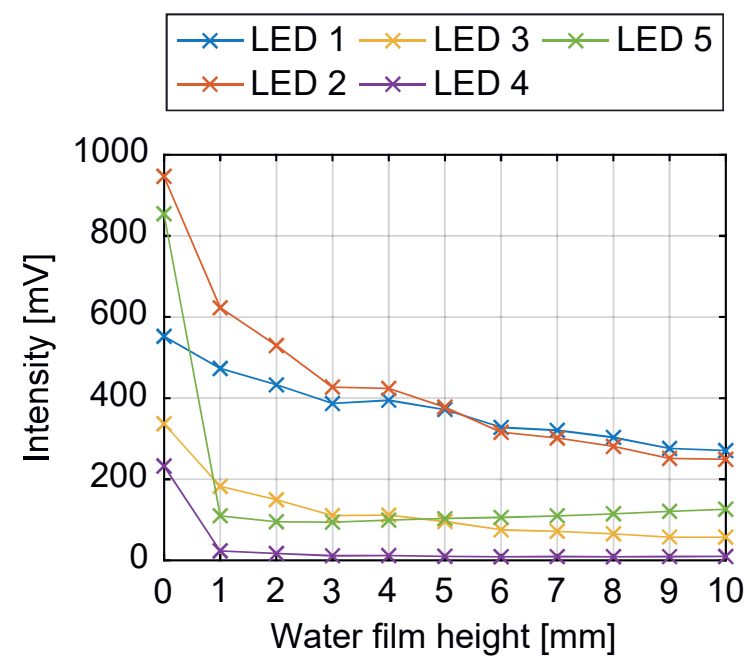

Fig. 7: Measurements of water film heights from $1 \mathrm{~mm}$ to $10 \mathrm{~mm}$ on a white surface. 
In general, the light with the shorter wavelengths $0.945 \mu \mathrm{m}, 1.2 \mu \mathrm{m}$ and $1.3 \mu \mathrm{m}$ decreases less than that of the longer wavelengths $1.45 \mu \mathrm{m}$ and $1.55 \mu \mathrm{m}$. This is due to the fact that light with the wavelengths $0.945 \mu \mathrm{m}$, $1.2 \mu \mathrm{m}$ and $1.3 \mu \mathrm{m}$ is more effectively transmitted by water and light with the wavelengths $1.45 \mu \mathrm{m}$ and $1.55 \mu \mathrm{m}$ is more strongly absorbed by water. By analyzing the progression with increasing water film height, it can be observed that the measurement at the water film height of $4 \mathrm{~mm}$ shows a slight measurement deviation. The intensity of LED 5 with a wavelength of $1.55 \mu \mathrm{m}$ increases from a water film height of $1 \mathrm{~mm}$. This increase in intensity suggests that the reflective component on the surface of the water is higher than the transmission and absorption component. As the distance between sensor and water surface decreases, the intensity values of LED 5 increase. For detailed analyses of the intensity change, the transmittance is formed. The transmittance $T$ is calculated for each wavelength $\lambda$ from the ratio of the intensities with water $I$ present to the intensity with a dry surface $I_{0}$. The following equation (4) shows the calculation of transmittance [11]

$$
T(\lambda)=\frac{I}{I_{0}} .
$$

Fig. 8 shows the transmittance for the respective wavelengths with increasing water film heights from $1 \mathrm{~mm}$ to $10 \mathrm{~mm}$ on the white surface. Since only discrete values can be recorded with the five LEDs, a diagram with discrete values is formed instead of an infrared spectrum.
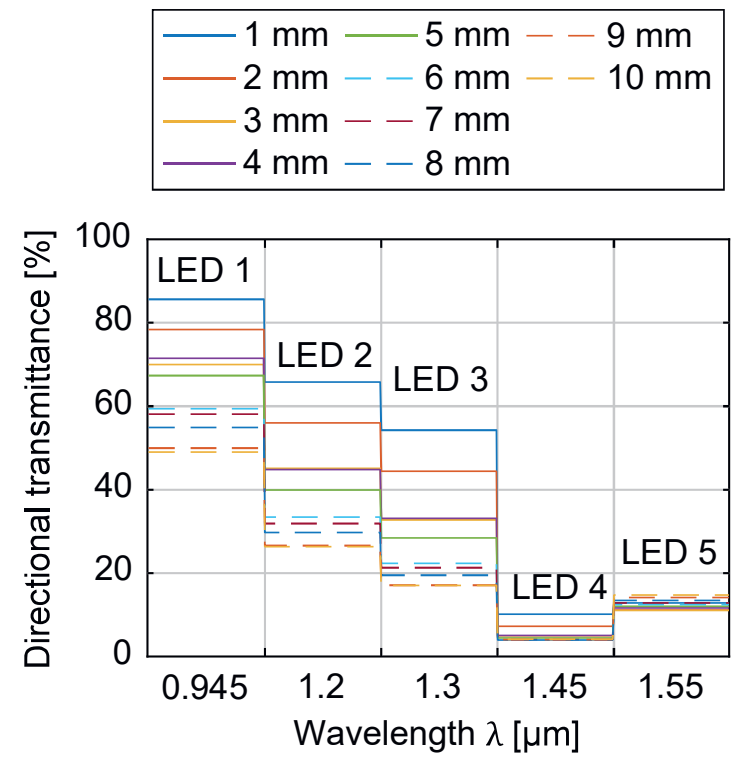

Fig. 8: Measured directional transmittance for increasing water film heights from $1 \mathrm{~mm}$ to $10 \mathrm{~mm}$ with white surface.
The following statements can be made with the help of the directional transmittance shown in Fig. 8. The discrete values of the transmittance at a water film height of $1 \mathrm{~mm}$ correspond approximately to those of the theory in Fig. 1. The transmittance is lowest at a wavelength of $1.45 \mu \mathrm{m}$. Subsequently, the transmittance increases for the wavelengths $1.55 \mu \mathrm{m}, 1.3 \mu \mathrm{m}$ and $1.2 \mu \mathrm{m}$ and the highest is at $0.945 \mu \mathrm{m}$.

A further statement refers to the resolution of the respective LEDs with increasing water film height. This is shown by the steps of the diagram with increasing water film height. The difference between the transmittances of two water film heights is approximately the same for the wavelengths $0.945 \mu \mathrm{m}, 1.2 \mu \mathrm{m}$ and $1.3 \mu \mathrm{m}$. By considering the transmittance, those wavelengths are suitable for quantitative statement about the water film height of $1 \mathrm{~mm}$ to $8 \mathrm{~mm}$. At a wavelength of $1.45 \mu \mathrm{m}$, the transmittance remains unchanged from a water film height of $3 \mathrm{~mm}$. The increase in intensity of the LED 5 with the wavelength of $1.55 \mu \mathrm{m}$ can be explained by the fact that the distance between sensor and surface of the water decreases with increasing water film height. Accordingly, the wavelengths $1.45 \mu \mathrm{m}$ and $1.55 \mu \mathrm{m}$ are suitable for a qualitative measurement of water film heights.

\section{Investigation of ice and water}

The aim of this measurement was to verify the detection of ice with the help of the theoretical approach. The water film height and the thickness of the ice layer on the white surface were $1 \mathrm{~mm}$. As the red solid line shows in Fig. 1, the wavelength of $1.5 \mu \mathrm{m}$ is strongly absorbed by ice. Thus, the classification of ice should be possible by comparing the different wavelengths.

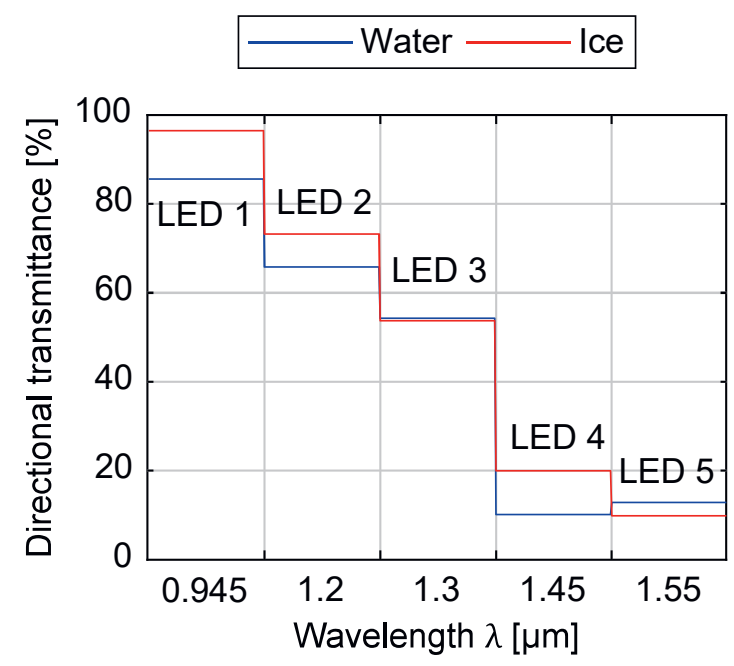

Fig. 9: $\quad$ Measured directional transmittance of water and ice. 
Fig. 9 shows that the intensities of LED 1 with a wavelength at $0.945 \mu \mathrm{m}$, LED 2 with a wavelength at $1.2 \mu \mathrm{m}$ and LED 4 with a wavelength at $1.45 \mu \mathrm{m}$ are absorbed more by water than by ice. The intensity of LED 3 with the wavelength at $1.3 \mu \mathrm{m}$ shows, for water and ice, almost the same transmittance. The theoretical approach to the detection of ice includes the comparison of the last two wavelengths. The wavelength at $1.45 \mu \mathrm{m}$ is more absorbed by water than a wavelength at $1.55 \mu \mathrm{m}$. Ice, on the other hand, transmits the wavelength at $1.45 \mu \mathrm{m}$ more efficiently and absorbs at $1.55 \mu \mathrm{m}$ more strongly. Other combinations such as LED 2 and LED 5 or LED 1 and LED 5 can also be used to distinguish between water and ice. Because here the transmittance of LED 1 and LED 2 increases like the transmittance of LED 4 when ice is present. Consequently, a distinction can be made between water and ice by comparing these wavelengths.

\section{Conclusion}

This work presented an infrared-based sensor system with one photodiode as the receiver and five different LEDs as the transmitter for contactless detection of wetness and ice on a surface. In the first part of this work, a theoretical approach was used to determine the appropriate emitters and receiving diodes. Five LEDs were selected as transmitter diodes. A photodiode in the wavelength range from $0.9 \mu \mathrm{m}$ to $1.7 \mu \mathrm{m}$ was selected as the receiver.

Subsequently, a measuring environment was created which reproducibly sprayed a defined water film onto a surface. This has a strain gage balance that measures the weight of the sprayed water to determine the water film height.

In the next step, the measurements were carried out. First, the influence of the brightness of the surface was investigated. A white and a black tile were used as the surface. The measurements showed that the white surface reflects much more light back than the black surface. The intensity of the monochromatic lights decreases from $55 \%$ to $72 \%$ depending on the wavelength compared to the black surface. With the help of the relative intensity the inversesquare law of distance could be shown. The intensity decreases by $75 \%$ when doubling the distance and is consequently only a quarter of the original intensity.

The measurements with water on the surface showed that the sensor system has a similar course as the transmission spectrum of water. Here it was evident that a part of the light of LED 5 with $1.55 \mu \mathrm{m}$ wavelength was absorbed by water and a part was reflected back from the water surface. Due to the intensity increased with the water film height, it is possible to measure the water film height with this sensor system.

The investigations carried out with ice showed that ice can be detected qualitatively by comparing different wavelengths. The light with a wavelength of $0.945 \mu \mathrm{m}, 1.2 \mu \mathrm{m}$ and $1.45 \mu \mathrm{m}$ is more strongly absorbed by water than by ice. For light with a wavelength of $1.55 \mu \mathrm{m}$ it is exactly the opposite. This sensor system is able to measure water film height and ice.

\section{References}

[1] Statistisches Bundesamt (Destatis), "Fachserie 8 Reihe 7 Verkehr Vekehrsunfälle," Wiesbaden, 2018.

[2] J. Döring, L. Tharmakularajah, J. Happel and K.-L. Krieger, "A novel approach for road surface wetness detection with planar capacitive sensors", J. Sens. Sens. Syst. 8, pp. 57-66 (2019); doi: 10.5194/jsss-8-572019

[3] R. Kurata, H. Watanabe, M. Tohno, T. Ishii and $\mathrm{H}$. Oouchi, "Evaluation of the detection characteristics of road sensors under poorvisibility conditions", IEEE Intelligent Vehicles Symposium, 2004, pp. 538-543 (2004); doi: 10.1109/IVS.2004.1336441

[4] F. Holzwarth and U. Eichhorn, "Non-contact sensors for road conditions, Sensors and Actuators", Sensors and Actuators A: Physical, 37-38, 121-127 (1993); doi: 10.1016/0924-4247(93)80023-A

[5] P. Jonsson, "Remote sensor for winter road surface status detection", SENSORS, 2011 IEEE, pp. 1285-1288 (2011); doi: 10.1109/ICSENS.2011.6127089

[6] O. Schrey, W. Brockherde and B. Hosticka, "Distance sensor and method for distance detection". Germany Patent US 20060214121 A1, 09052005.

[7] F. Klempau, "Untersuchungen zum Aufbau eines Reibwertvorhersagesystems im fahrenden Fahrzeug", Darmstadt: Technische Universität Darmstadt, 2003.

[8] S. G. Warren, „Optical constants of ice from the ultraviolet to the microwave", Appl. Opt., 23, 1206-1225 (1984); doi: 10.1364/AO.23.001206

[9] U. Harten, „Physik“, Berlin Heidelberg: Springer-Verlag, (2014); doi: 10.1007/9783-642-53854-4

[10] L. Tharmakularajah, J. Döring and K.-L. Krieger, "Infrared-based determination of the type and condition of the road surface" in 19. Internationales Stuttgarter Symposium Automobil- und Motorentechnik, Stuttgart, 2019.

[11] H. Günzler and H.-U. Gremlich, „IR-Spektroskopie“, Weinheim: WILEY-VCH Verlag $\mathrm{GmbH}$ \& Co. KGaA, (2003); doi: $10.1002 / 9783527662852$ 